\title{
Time at the center, or time at the side? Assessing current models of time perception
}

\author{
Joachim Hass ${ }^{1}$ and Daniel Durstewitz ${ }^{2}$ \\ ${ }^{1}$ Dept. of Mathematics and Statistics, Boston University, Boston, MA 02215, USA \\ ${ }^{2}$ Dept. Theoretical Neuroscience, Bernstein-Center for Computational Neuroscience, \\ Central Institute of Mental Health, Medical Faculty Mannheim of Heidelberg University, \\ Mannheim, Germany
}

\begin{abstract}
The ability to tell time is a crucial requirement for almost everything we do, but the neural mechanisms of time perception are still largely unknown. One way to approach these mechanisms is through computational modelling. This review provides an overview of the most prominent timing models, experimental evidence in their support, and formal ways for understanding the relationship between mechanisms of time perception and the scaling behavior of time estimation errors. Theories that interpret timing as a byproduct of other computational processes are also discussed. We suggest that there may be in fact a multitude of timing mechanisms in operation, anchored within areaspecific computations, and tailored to different sensory-behavioral requirements. These ultimately have to be integrated into a common frame (a "temporal hub") for the purpose of decision making. This common frame may support Bayesian integration and generalization across sensory modalities.
\end{abstract}

\section{Introduction}

Processing of temporal information is so ubiquitous and central in our daily life that we rarely notice the ease with which we differentiate between stimuli with tiny differences in time, such as the syllables $/ \mathrm{ba} /$ and $/ \mathrm{pa} /$ in speech, or coordinate dynamic sensory information with complex movements to catch a ball just in the right moment. How the brain manages to process temporal information in such precision and in the face of vastly different stimuli is still a mystery. Curiously, this enigma is not due to a shortage of theoretical proposals about the mechanisms of time perception - during the last two decades, the number of theoretical studies on time perception has exploded, leaving one with the impression that basically any time-varying process in the brain could be used to estimate duration.

In the current review, we argue that the field of time perception has now matured to a degree where it is possible to classify timing models into a number of candidate mechanisms which can be evaluated by examining the most established of experimental results. Furthermore, we show how current findings point toward the existence of multiple neural mechanisms of time perception, arising possibly as a byproduct of other computations. We conclude by discussing how these diverse representations are integrated into a single, coherent estimate of duration as required for decision making and to guide behavioral output.

\section{Classes of time perception models}

The models of time perception which are most frequently discussed in the current literature can be classified into four principle neural mechanisms [1] (Figure 1): 
1. Ramping activity models (Figure 1, upper left) assume that time is estimated by means of slowly increasing firing rates, peaking at the end of the interval to be estimated $[2 *, 3,4]$. By changing the slope of the ramp (as illustrated by the dashed black line in Figure 1), different intervals $\left(T_{\text {stop }}-T_{\text {start }}\right)$ can be encoded. This mechanism is probably best supported by electrophysiological studies, as ramping activity has been found in many brain regions and correlates with behavioral estimates of time $[5,6]$.

2. In delay line models (Figure 1, upper right), activity spreads across sequentially arranged pools of neurons (represented by the green ellipses in Figure 1), with a relatively constant delay time between pools, and the interval duration is encoded by the location of the activity bump along that sequence (filled circles in Figure 1). One specific physiological implementation of this mechanism could be a "synfire chain" $[7,8]$, but another, related, implementation would be a bank of neural elements with different time constants which effectively perform a kind of spectral decomposition of the stimulus $[9,10,11]$.

3. Neural oscillator models (Figure 1, lower right) employ cortical oscillations in different frequency bands (colored circles in Figure 1), either by counting the number of revolutions during an interval (the highly influential pacemaker-accumulator concept $[14,15]$ ), or by detecting coincident events within a bank of oscillators of similar frequency, encoding longer intervals by the "beat" (the "striatal beat model" $[16,17,18,19,20]$ which is based on striatal anatomy).

4. In state-dependent timing models (Figure 1, lower left), a specific duration is not encoded through a purpose-tailored neural mechanism, but rather through the temporal evolution of the collective state of the entire network (represented in Fig. 1 as a trajectory through a state space spanned by the firing rates (FR) of three neurons), exploiting recurrence and naturally occurring variation in neural and synaptic properties $[12 *, 13]$.

From a theoretical point of view, all of these mechanisms can be understood within the common language of dynamical systems theory: Collecting all the dynamical variables of the system (e.g., neural firing rates, state of synaptic currents, etc.) into one state vector, the temporal evolution of the system can be described as a trajectory in a high-dimensional state space spanned by these variables (Figure 1D). Knowing where in state space the system was located when a triggering stimulus occurred, the position along that trajectory encodes information about the time that has passed since stimulus presentation. The state-dependent timing model makes this idea most explicit, but formally any of the other models described above can be represented in this format: The ramping activity models through the relevant set of neural firing rate variables, the synfire chain models in the space of constituent pools or units, and the oscillator models in terms of neural phase variables. While the state-dependent models leave the exact mechanism often open and claim that anything can be exploited for timing purposes with proper readout, the other models may be seen as imposing specific constraints on the state space, form of trajectory, and mapping of time on trajectory.

\section{Experimental constraints on time perception models}

To assess whether these canonical timing models provide reasonable candidate mechanisms for interval time estimation in the brain, it is important to test whether they can reproduce central experimental findings. One constraint is that we should give more credibility to models which are compatible with physiological observations. All four of the models discussed above are, however, at least tentatively in agreement with one bit of physiology or another. We also consider two other prominent and often replicated sets of evidence in evaluation of these models, the psychophysical laws of time perception and the susceptibility of subjective time to manipulations in neurotransmitter systems (e.g., dopamine) and other stimulus properties. The psychophysical observations are that both subjective duration and its variability increase linearly with the duration of an interval. The linear increase in variability is known as Weber's law, or the scalar property, and 
has been proposed to be a universal property of time perception. While observed in many experimental settings, there are also numerous violations of Weber's law $[8,15]$, in particular for very long and very short intervals. The classic state-dependent networks and ramping activity models have not yet been tested in this regard, although a recent modelling study exploits stochastic ramping and finds the scalar property to hold [4]. Among the delay line models, the synfire chain has been shown to approximate the scalar property in a limited range of intervals, and to also reproduce the deviations from this law at longer and shorter intervals [8]. Spectral models $[9,10,11]$ account for the scalar property by means of the specific set of filters included in the model: As equal spacing is assumed in frequency space, shorter times are naturally more finely sampled compared to longer ones. Finally, in classical oscillator models (such as scalar expectancy theory [21] and the striatal beat model [16]), the scalar property was imposed by explicitly adding a scalar source of noise, so that the mechanistic origin of the scalar property itself remains unclear. In a more recent analysis of the striatal beat model [18], scalar timing emerges from the equal spacing of frequencies, similar to those used in spectral models. Apart from these approximate and rather explicit ways to impose the scalar property, there is also a class of processes that inherently exhibits scalar timing (see below).

Regarding the distortion of subjective duration, an impressive body of literature has shown that increased levels of dopamine, and in particular the activation of D2-type dopamine receptors, prolong interval time estimates in both humans and animals, as if "speeding up" an internal clock. The opposite effect is observed when dopamine levels or D2 receptor activation are diminished $\left[22,23,24^{*}, 25\right]$ (but see $\left[26^{* *}, 27\right]$ for the role of D1 receptors). So far, only few attempts have been made to explain this dopaminergic modulation of time perception. In a recent extension of the striatal beat model, it was explicitly assumed that dopamine modulation would change the frequencies of the oscillators [17]. In contrast, preliminary results in ramping activity models indicate that the dopaminergic modulation of interval estimates could emerge intrinsically from the experimentally measured dopamine effects on synaptic conductances [28]. Moreover, similar to the direct effects of neuromodulators on interval estimation, stimulus attributes that may engage neuromodulatory systems, like intense, arousing, or attention provoking stimuli, have also been shown to cause an overestimation of intervals [29]. Although such experimental manipulations provide an interesting testbed for timing models, so far they have hardly been explored in modelling studies.

\section{Current theoretical developments}

In addition to the specific model proposals discussed above, three other theoretical themes have emerged in recent years:

\section{Timing from noise and the origin of the scalar property}

In classical timing models (Figure 1), noise was basically seen as a nuisance which impedes accurate interval time estimation generated by an underlying deterministic process. However, random variability could itself be exploited as a timing mechanism. To illustrate this, consider a drop of ink dropped into a pond of water. As time goes by, thermal fluctuations cause the ink spot to expand, allowing for an estimation of elapsed time from the radius of the ink area which reflects the noisedriven diffusion process. Along this line, some earlier models proposed that interval time estimates may derive from the average decay time in a set of bistable units with noise causing switches from one to the other stable state [30]. More recently, several models have been developed that exploit random fluctuations in neural responses [31,4] or external stimuli [32]. Interestingly, all these noisebased models reproduce the scalar property. A recent information-theoretical analysis [33*] found that this is not a coincidence - a broad class of timing models that relies on changing variability were shown to naturally adhere to this law. If systematic (average) changes are used for timing, on the other hand, as it is the case for most classical models, variability increases sub-linearly, while models that rely on decaying similarities (correlations) between stimulus patterns over time (e.g. [32]) show 
a super-linear increase of variability. This analysis has interesting consequences for the assessment of time perception models, as all three scaling behaviors (sublinear, linear and superlinear) have indeed been observed empirically under different circumstances, for instance at different time scales [34]. This raises the possibility that fundamentally different principle mechanisms may operate in different temporal regimes.

\section{Timing as a byproduct of other computational processes}

Most time perception models have been designed with the explicit purpose to encode time. However, many of the processes that are used for time perception could also be used for other computational processes, or, vice versa. Recent experimental evidence suggests that timerepresenting mechanisms are active even in tasks where timing is not explicitly required in any wayperhaps not surprisingly, as an animal can never be sure which predictive relationships involving time may become important in the future. For instance, when Romo and colleagues [35] let monkeys discriminate tactile stimuli, activity in the premotor cortex showed ramping firing rates which peaked at the time of the decision. Although these rate changes also encoded the decision outcome, the time-dependent ramping explained a larger amount of variability in the firing rates. Similarly, a recent analysis of prefrontal cortex activity during a range of different tasks in different environments showed that elapsed time was accompanied by systematic drift in the neural population state that accounted for a large proportion of variability in activity patterns [36*] (Figure 2), although timing was not a crucial ingredient of the task. The recently discovered "time cells" in hippocampus [37] can also be seen to encode time as a byproduct of other computations, such as motion [38*] or the recall of odor memories [39]. Indeed, timing may be such an inherent property of most neuro-computational processes, from the perception of time-varying signals all the way up to behavioral planning, that its neural underpinnings may be intimately intertwined with the unfolding of the computational dynamics. State-dependent timing models (Figure 1D) naturally blend in with this idea, as they point out that any trajectory in state space produced by a network's computational dynamics also provides an encoding of time.

\section{Timing as Bayesian inference}

As timing could arise from different mechanisms and within different sensory and motor domains, the question arises how different sources of temporal information are combined into a unique, coherent representation of time that can be used in decision making processes. Recently, a number of studies combined psychophysical experiments with computational modelling to show that the brain may derive and integrate time estimates in a Bayesian manner $\left[40,41^{*}, 42,43,44,45,46\right]$, implying that temporal stimuli from different sources are weighted by their relative precision (inverse variability). In this way, conflicting temporal information, e.g. from auditory and visual sources, is resolved by giving more weight to the more precise source (which is usually audition $[43,46]$, but see [42]). Bayesian computation requires the brain to keep representations of probability distributions, either explicitly or in parameterized form $[47,48]$. Shi, Church and Meck recently made an attempt to map these different elements of Bayesian computation onto the elements of the classical pacemaker-accumulator framework [45].

\section{Time at the center, or time at the side?}

The findings above may shed new light on the old question of whether time is represented by a centralized "internal clock" or rather by a network of different time processors distributed throughout the brain $[49,50,15]$. The multitude of possible timing mechanisms (potentially associated with different scaling laws) and the findings and ideas on timing as a byproduct of other computational processes suggest a distributed representation of time. It seems likely that these timing processes are closely integrated with other computations that are performed in the respective area $\left[36^{*}, 38^{*}\right]$, where population states encoding a specific interval may at the same time also encode a certain odor memory [39] or spatial environment [36*]. This integrated view of 
computation and timing may also provide a parsimonious explanation for how and why subjective time is influenced by other, non-temporal factors, like attention: Any change in the computational process, reflected in the course and velocity along a dynamical system's trajectory [51], would automatically also change the encoding of time, thus leading to the observed distortions in estimated duration.

On the other hand, numerous studies show that the different temporal representations are not independent but are integrated in a potentially Bayesian-optimal way. Thus, there must be some kind of interaction among the timing circuits in different brain areas. This may be in terms of direct, mutual synaptic interactions, but ultimately, for the purpose of decision making and generating behavioral outputs, there may also be some convergence onto a uniform time representation. This convergence may be implemented in terms of the anatomical projections from computationally specific time-processing areas onto those brain structures involved in decision making, such as the prefrontal cortex and the striatum. Thus, one may assume that Bayesian integration of temporal information occurs at this processing stage, which thereby provides a kind of "temporal hub" [42] (Figure 3). The synaptic weights from the different brain regions providing the individual time representations onto the read-out hub could weight their respective outputs in a Bayesian way, i.e. according to their relative precision. Mechanistically, one way to achieve this could be a learning rule that combines spike timing-dependent plasticity with homeostatic synaptic scaling. It has been previously shown that such a learning rule can impose competition between different representations of time based on their temporal precision [8]. Since ultimately, when it comes down to deciding the future course of actions, also in non-temporal domains sensory information from various sources has to be integrated, one may speculate whether such a kind of 'hub' is a more general entity which functionally and anatomically overlaps with the temporal hub.

The hypothesis of an effective integrated time representation within the decision network also provides a natural explanation for temporal generalization that has been observed in learning studies: As summarized in a recent review [52*], it is possible to improve performance (i.e. reduce variability) in timing tasks by training over several days, and this improvement generalizes to stimuli from other modalities (e.g. from audition to vision, or between auditory stimuli of different frequencies), and even from perception to motion [53], but not to other intervals than the one trained for. This could be explained through plastic changes both within the temporal hub, and between the hub and the individual timing networks: Repeated presentation of a $300 \mathrm{~ms}$ auditory stimulus activates a particular set of neurons in the hub (upper right two neurons in Figure 3 ) and strengthens both the synapses from the auditory network to the hub and those within the hub. Assuming that hub neurons which encode for the same duration share some random synaptic connections, a presentation of a $300 \mathrm{~ms}$ visual interval will profit from the stronger synapses within the temporal hub, leading to the generalization effect. In particular, assuming that strengthening of overlapping connections between two hub populations will require repeated exposures, this framework predicts that generalization would only occur later during training, once the auditory-tovisual synaptic links are strong enough for the former to drive the latter. Such a delay in generalization has been confirmed in recent learning study [54].

\section{Conclusion}

In summary, we propose that the representation of interval time may be an inherent property or "byproduct" of other computations performed by various brain areas. As such, there may be (almost) as many specific timing mechanisms in the brain as there are different computational domains. Yet, these may fall into a few different canonical classes (timing from systematic drift, timing from variability, etc.) which give rise to different forms of psychophysical scaling behavior. A unifying theoretical framework may be provided by dynamical systems theory (in the form of stochastic differential or difference equations, incorporating sources of random variability), according to which 
computational processes can be understood in terms of trajectories meandering through a highdimensional state space $\left[55^{* *}, 56\right]$. Any such trajectory provides an implicit representation of the passage of time, therefore providing a natural and mechanistic account of how other computational processes and timing go hand in hand. At the same time, timing information from these many different sources would ultimately have to be combined in the process of decision making to yield a unique behavioral response. The evidence reviewed above suggests that this integration may happen in a Bayesian optimal way.

\section{Acknowledgments}

This work was supported by grants from the German ministry for education and research (BMBF, 01GQ1003B) and the Deutsche Forschungsgemeinschaft to DD (Du 354/6-1 \& 7- 2). JH was supported by a grant from the Army Research Office (ARO) of the American Department of Defense (W911NF-141-0374).

\section{References}

1. Hass J, Durstewitz D: Neurocomputational Models of Time Perception. Adv Exp Med 2014, 829:49-71.

2. * Durstewitz D: Self-organizing neural integrator predicts interval times through climbing activity. J Neurosci 2003, 23:5342-5353.

First paper to introduce the idea of representing arbitrary interval times dynamically by means of detuning a neural integrator, and its putative relation to climbing activity in cortex.

3. Durstewitz D: Neural representation of interval time. Neuroreport 2004, 15(5):745-749.

4. Simen P, Balci F, Cohen JD, Holmes P: A model of interval timing by neural integration. J Neurosci 2011, 31:9238-9253.

5. Matell MS, Shea-Brown E, Gooch C, Wilson AG, Rinzel J: A heterogeneous population code for elapsed time in rat medial agranular cortex. Behav Neurosci 2011, 125:54-73.

6. Wittmann M: The inner sense of time: how the brain creates a representation of duration. Nat Rev Neurosci 2013, 14:217-23.

7. Kitano $\mathrm{K}$, Okamoto $\mathrm{H}$, Fukai $\mathrm{T}$ : Time representing cortical activities: two models inspired by prefrontal persistent activity. Biol Cybern 2003, 88(5):387-394.

8. Hass J, Blaschke S, Rammsayer T, Herrmann JM: A neurocomputational model for optimal temporal processing. J Comput Neurosci 2008, 25(3):449-464.

9. Grossberg S: Adaptive resonance theory: How a brain learns to consciously attend, learn, and recognize a changing world. Neural Networks 2012, 37:1-47.

10. Shankar KH, Howard MW (2012): A scale-invariant internal representation of time. Neural Computation 2012, 24(1):134-193.

11. Howard MW, MacDonald CJ, Tiganj Z, Shankar KH, Du Q, Hasselmo ME, Eichenbaum H: A unified mathematical framework for coding time, space, and sequences in the hippocampal region. $J$ Neurosci 2014, 34(13):4692-4707.

12. * Buonomano DV: Decoding temporal information: a model based on short-term synaptic plasticity. J Neurosci 2000, 20(3):1129-1141.

Introducing the idea of representing time by phase-space trajectories.

13. Buonomano DV, Laje R: Population clocks: motor timing with neural dynamics. Trends Cogn Sci 2010, 14:520-27.

14. Allman MJ, Teki S, Griffiths TD, Meck WH: Properties of the internal clock: first-and secondorder principles of subjective time. Annu Rev Psychol 2014, 65:743-771. 
15. Grondin S: About the (non) scalar property for time perception. Adv Exp Med 2014, 829:17-32.

16. Matell MS, Meck WH: Cortico-striatal circuits and interval timing: coincidence detection of oscillatory processes. Cogn Brain Res 2004, 21(2):139-170.

17. Oprisan SA, Buhusi CV: Modeling pharmacological clock and memory patterns of interval timing in a striatal beat-frequency model with realistic, noisy neurons. Front Integr Neurosci 2011, 5:52.10.3389/fnint.2011.00052.

18. Oprisan SA, Buhusi: What is all the noise about in interval timing? Philos Trans $R$ Soc Lond $B$ Biol Sci 2014, 369:20120459.

19. Meck WH, Penney TB, Pouthas V: Cortico-striatal representation of time in animals and humans. Curr Opin Neurobiol 2008, 18:145-52.

20. Merchant $\mathrm{H}$, Harrington DL, Meck WH: Neural basis of the perception and estimation of time. Annu Rev Neurosci 2013, 36:313-336.

21. Gibbon J: Ubiquity of scalar timing with a Poisson clock. J Math Psychol 1992, 36(2):283-293.

22. Meck WH, Cheng RK, MacDonald CJ, Gainetdinov RR, Caron MG, Cevik MÖ: Gene-dose dependent effects of methamphetamine on interval timing in dopamine-transporter knockout mice. Neuropharmacology 2012, 62:1221-29

23. Heilbronner SR, \& Meck WH: Dissociations between interval timing and intertemporal choice following administration of fluoxetine, cocaine, or methamphetamine. Behav Proc 2014, 101:123-134.

24. * Wiener M, Lohoff FW, Coslett HB: Double dissociation of dopamine genes and timing in humans. J Cogn Neurosci 2011, 23:2811-21.

Demonstration of dopaminergic effects on time perception in humans by means of dissociating different dopamine-related genes according to their locus of action (prefrontal cortex vs. striatum).

25. Coull JT, Hwang HJ, Leyton M, Dagher A: Dopamine precursor depletion impairs timing in healthy volunteers by attenuating activity in putamen and SMA. J Neurosci 2012, 32:16704-15.

26. ** Narayanan NS, Land BB, Solder JE, Deisseroth K, DiLeone RJ: Prefrontal D1 dopamine signaling is required for temporal control. Proc Natl Acad Sci USA 2012, 109(50):20726-20731. First study to show direct involvement of D1 receptors in time perception in the prefrontal cortex.

27. Parker KL, Alberico SL, Miller AD, Narayanan NS: Prefrontal D1 dopamine signaling is necessary for temporal expectation during reaction time performance. Neuroscience 2013, 255:246-254.

28. Hass, J, Durstewitz D: Models of dopaminergic modulation. Scholarpedia 2011, 6(8):4215.

29. Matthews WJ, Terhune DB, van Rijn H, Eagleman DM, Sommer MA, Meck WH: Subjective duration as a signature of coding efficiency: Emerging links among stimulus repetition, prediction coding, and cortical GABA levels. Timing Time Percept Rev 2014, 1:1-9.

30. Okamoto H, Fukai T: Neural mechanism for a cognitive timer. Phys Rev Lett 2001, 86:3919-3922.

31. Almeida R, Ledberg A: A biologically plausible model of time-scale invariant interval timing. J Comput Neurosci 2010, 28:155-175.

32. Ahrens MB, Sahani M: Observers exploit stochastic models of sensory change to help judge the passage of time. Curr Biol 2011, 21(3):200-206.

33. * Hass J, Herrmann JM: The neural representation of time: An information-theoretic perspective. Neural Comput 2012, 24(6):1519-1552. Information-theoretical framework that links different scaling laws of timing errors with fundamentally different timing mechanisms.

34. Gibbon J, Malapani C, Dale CL, Gallistel CR: Toward a neurobiology of temporal cognition: advances and challenges. Curr Opin Neuro 1997, 7:170-184. 
35. Romo R, Hernández A, Zainos A: Neuronal correlates of a perceptual decision in ventral premotor cortex. Neuron 2004, 41(1):165-173.

36. * Hyman JM, Ma L, Balaguer-Ballester E, Durstewitz D, Seamans JK (2012). Contextual encoding by ensembles of medial prefrontal cortex neurons. Proc Natl Acad Sci USA 2012, 109(13):50865091.

Shows that time is encoded in prefrontal cortical activity patterns, in ways different from hippocampal time encoding, although time perception was not an explicit requirement of the tasks.

37. MacDonald CJ: Prospective and retrospective duration memory in the hippocampus: is time in the foreground or background? Philos Trans R Soc Lond B Biol Sci 2014, 369(1637):20120463.

38. * MacDonald CJ, Lepage KQ, Eden UT, Eichenbaum H: Hippocampal "time cells" bridge the gap in memory for discontiguous events. Neuron 2011, 71:737-749.

First report of "time cells" in the hippocampus, which encode time as a byproduct of other computations, although timing was not explicitly required for the task.

39. MacDonald CJ, Carrow S, Place R, Eichenbaum H: Distinct hippocampal time cell sequences represent odor memories in immobilized rats. J Neurosci 2013, 33:14607-14616.

40. Acerbi L, Wolpert DM, Vijayakumar S: Internal representations of temporal statistics and feedback calibrate motor-sensory interval timing. PLoS Comput Biol 2012, 8(11):e1002771.

41. * Cicchini GM, Arrighi R, Cecchetti L, Giusti M, Burr DC: Optimal encoding of interval timing in expert percussionists. J Neurosci 2012, 32:1056-60

Provides evidence for a Bayesian framework of time perception by comparing accuracy and precision of time estimates between drummers, string players and non-musicians.

42. Hass J, Blaschke S, Herrmann JM: Cross-Modal Distortion of Time Perception: Demerging the Effects of Observed and Performed Motion. PLOS ONE 2012, 7(6):e38092.

43. Burr D, Della Rocca E, Morrone MC: Contextual effects in interval-duration judgements in vision, audition and touch. Exp Brain Res 2013, 230(1):87-98.

44. Sohn $\mathrm{H}$, Lee $\mathrm{SH}$ : Dichotomy in perceptual learning of interval timing: calibration of mean accuracy and precision differ in specificity and time course. J Neurophysio/ 2013, 109:344-362.

45. Shi Z, Church RM, Meck WH: Bayesian optimization of time perception. Trends Cogn Sci 2013, 17(11):556-564.

46. Hartcher-O'Brien J, Di Luca M, Ernst MO (2014). The duration of uncertain times: audiovisual information about intervals is integrated in a statistically optimal fashion. PLOS ONE 2014, 9(3):e89339.

47. Yang T, Shadlen MN: Probabilistic reasoning by neurons. Nature 2007, 447(7148):1075-1080.

48. Pouget A, Beck JM, Ma WJ, Latham PE: Probabilistic brains: knowns and unknowns. Nat Neurosci 2013, 16(9):1170-1178.

49. Buonomano DV, Karmarkar UR: How Do We Tell Time? Neuroscientist 2002, 8(1):42-51.

50. Ivry RB, Schlerf JE: Dedicated and intrinsic models of time perception. Trends Cogn Sci 2008, 12(7):273-280.

51. Lapish CC, Balaguer-Ballester E, Seamans JK, Phillips AG, Durstewitz D (2015). Amphetamine exerts dose-dependent changes in prefrontal cortex attractor dynamics during working memory. J Neurosci 2015, 35(28).10172-10187.

52. Bueti D and Buonomano DV: (2014) Temporal Perceptual Learning. Timing Time Percept (2014), 2:261-289.

53. Meegan DV, Aslin RN, Jacobs RA: Motor timing learned without motor training. Nat Neurosci 2000, 3(9):860-862. 
54. Wright BA, Wilson RM, Sabin AT: Generalization lags behind learning on an auditory perceptual task. J Neurosci 2010, 30:11635-11639.

55. ** Karmarkar UR, Buonomano DV: Timing in the absence of clocks: encoding time in neural network states. Neuron 2007, 53(3):427-438.

Experimental test of the state-dependent timing model using psychophysical experiments in humans, confirming dependence of time estimates on previous temporal information, as predicted by the model.

56. Durstewitz D, Deco G: Computational significance of transient dynamics in cortical networks. Eur J Neurosci 2008, 27(1):217-227. 


\section{Figure 1}

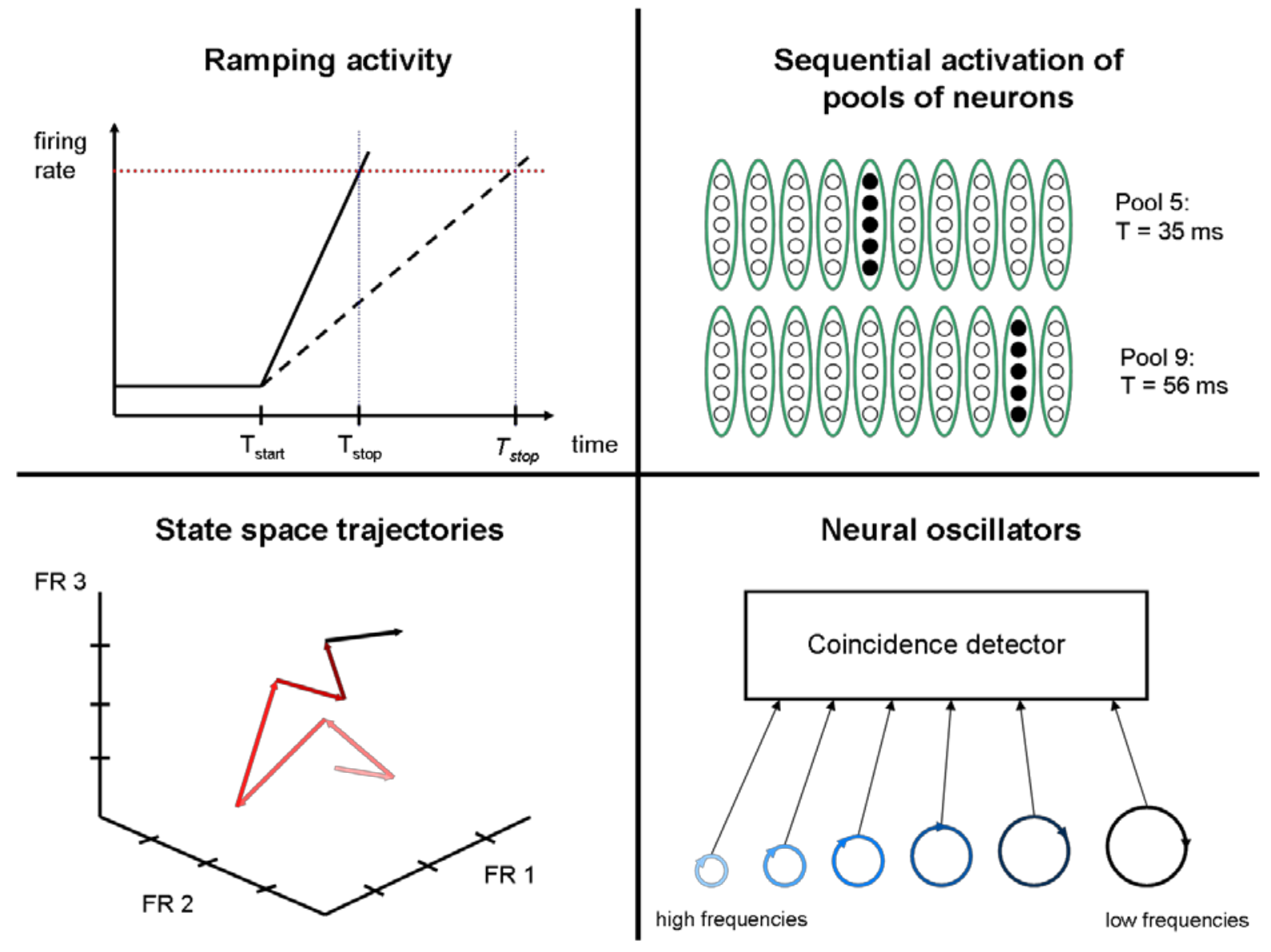

Figure 1: Illustration of four canonical classes of timing models as discussed in the text. With kind permission from Springer Science+Business Media: Advances in Experimental Medicine,

"Neurocomputational Models of Time Perception", 829, 2014, 49-71, J. Hass and D. Durstewitz, Figure 2. 


\section{Figure 2}
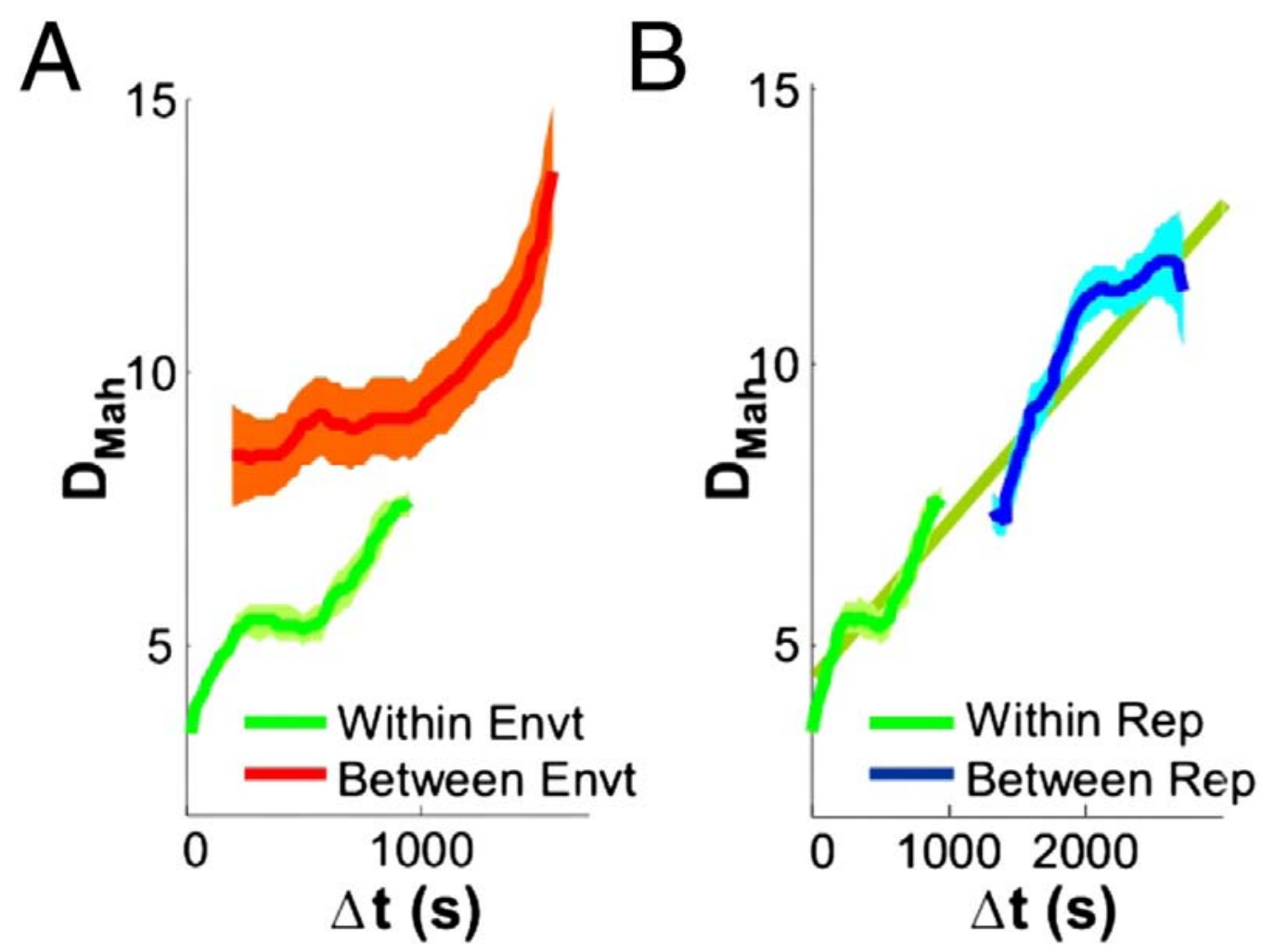

Figure 2: Impact of temporal and environmental context on neural ensemble activity in the rat medial prefrontal cortex. Both graphs depict the Mahalanobis distance $\left(D_{\text {Mah }}\right)$ between neural population states as a function of their separation in time $(\Delta t)$. The Mahalanobis distance is a kind of statistical distance measure that takes the data scatter into account. (A) For one specific example session, $D_{\text {Mah }}$ is plotted as a function of $\Delta t$, indicating a systematic drift of the population state with the passage of time both within the same environment (green curve) or across different environments (red curve). When the animal is moved from one environment into another (red vs. green curve), the neural population state jumps to a new position (leading to a sudden increase in distance), but otherwise continues its systematic drift with time. (B) If the same environmental setting is repeatedly presented, the increase in spatial separation of population vectors across repetitions as measured by $D_{\text {Mah }}$ can be explained largely by the passage of time: $A$ linear regression model fitted to the data in A only (that is, only to the first environmental presentations) yields a reasonable prediction for the neural state separation upon the second repetition of the same environment. Shaded areas = SEM. Reproduced with permission from Hyman et al. 2012. 


\section{Figure 3}

auditory modality $\quad \cdots \quad$ visual modality

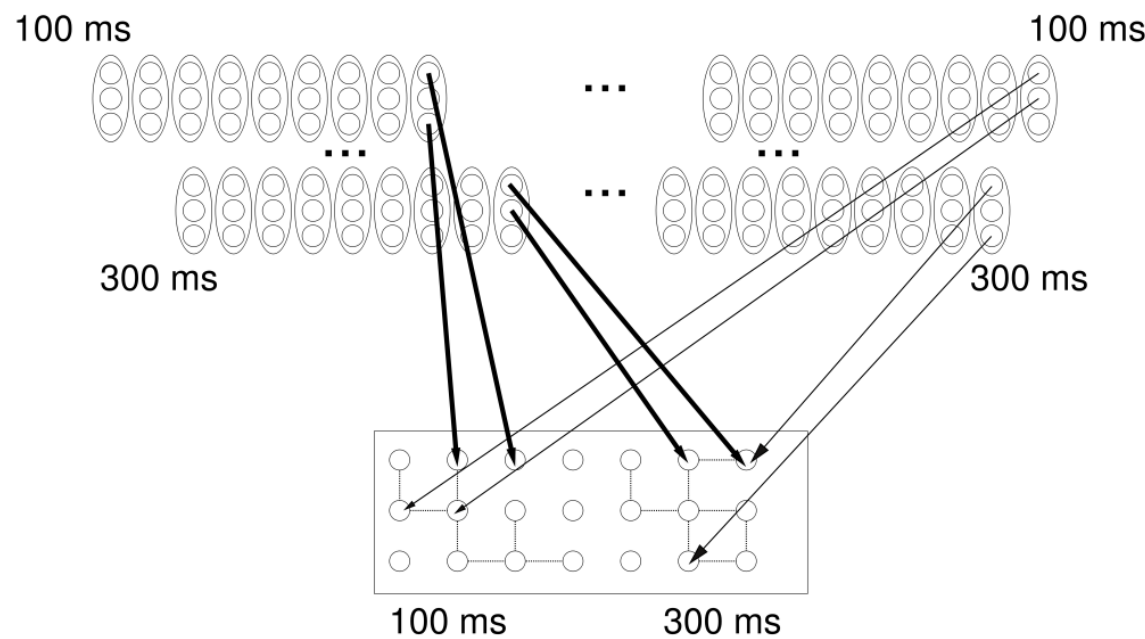

multiple temporal processes

temporal hub

Figure 3: Illustration of a possible integration of temporal information from different sensory modalities into a "temporal hub" within the brain's decision network. In the top part, multiple timing processes from different modalities are shown, illustrated as synfire chains for sake of example. The lower part depicts the temporal hub, a recurrent network onto which the modality-specific timing networks project. The synaptic weights (illustrated by the strength of the arrows) are determined by the reliability of each source. Here the case is illustrated where auditory processes carry more temporal information than visual ones. Dotted lines indicate recurrent connections within the hub which form cell assemblies for different intervals (100 and $300 \mathrm{~ms}$ in this example). 